\title{
Atomic entanglement sudden death in a strongly driven cavity QED system
}

\author{
Ying-Jie Zhang, Zhong-Xiao Man and Yun-Jie Xia * \\ Shandong Provincial Key Laboratory of Laser Polarization and Information Technology, \\ Department of Physics, Qufu Normal University, \\ Qufu 273165, Peoples Republic of China
}

\begin{abstract}
We study the entanglement dynamics of strongly driven atoms off-resonantly coupled with cavity fields. We consider conditions characterized not only by the atom-field coupling but also by the atom-field detuning. By studying two different models within the framework of cavity QED, we show that the so-called atomic entanglement sudden death (ESD) always occurs if the atom-field coupling larger than the atom-field detuning, and is independent of the type of initial atomic state.

PACS numbers: 03.67.Mn, 42.50.Dv, 03.65.Ud
\end{abstract}

Keywords: entanglement dynamics, entanglement sudden death, off-resonance

\footnotetext{
* The corresponding author. Email address: yjxia@mail.qfnu.edu.cn
} 


\section{Introduction}

Entanglement plays a crucial role in quantum information processing [1]. Quantum algorithms (particularly, in Shor's algorithm, to find the prime factors of an $n$-bit integer) exploit entanglement to speed up computation. Entanglement dynamics have been a difficult subject and have attracted extensive interest recently ranging from two-qubit systems [2 -5], to continuous variables $[6,7]$, spin systems $[8,9,10]$, and multi-partite systems $[11-14]$. Entanglement of open quantum systems also has attracted considerable attention due to its significance for both fundamentals and applications of quantum information processing $[15,16]$. Such as, the paper [15] gives us the non-Markovian entanglement evolution of two uncoupled qubits which are coupled to their own independent environments. Origination and survival of entanglement for two independent qudits dephasingly coupled to a common zero-temperature, super-Ohmic, bosonic environment is studied in [16]. Moreover, in the close systems, proposals [17, 18] have been made for the direct measurement of finite-time disentanglement in cavity QED, and real-time detection of entanglement sudden death (ESD) has been reported very recently[19, 20,21].

The interaction of a two-level atom with a quantized single mode of a harmonic oscillator, was called the Jaynes-Cummings (JC) model [22]. The JC model has found its natural playground in the field of cavity quantum electrodynamics (CQED), and extensions of the JC model to more atoms and more modes, externally driven or not, have been developed. Presently, we enjoy a vast number of theoretical and experimental developments. In [19], the authors dealt with a double JC model in which two initially entangled two-level atoms $A$ and $B$ are independently coupled with separate cavity fields $a$ and $b$ respectively, but there are no interactions at all between the subsystems $A a$ and $B b$. Focusing on the atomic subsystem they found that depending on the type of initial state of atoms $A B$, their entanglement may or may not exhibit ESD. From [19], we can acquire that the authors have taken Bell-like states as the initial state of atoms and assumed the initial cavity field to be in vacuum. As a consequence, ESD was found to be sensitive to the initial atomic state. That is to say, ESD may occur for a certain type of initial atomic state but does not appear for another type.

In this paper, we consider a system consisting of a two-level atom trapped inside a single mode cavity. The atom is additionally driven by a strongly classical field. The experimental implementation seems to be feasible due to the recent advances in deterministic trapping of atoms in the optical cavities $[23,24]$. We show that under-taking atomic pure Bell-like states 
$\left|\Phi_{A B}\right\rangle=\cos \theta\left|e_{A}, e_{B}\right\rangle+\sin \theta e^{i \phi}\left|g_{A}, g_{B}\right\rangle$ or $\left|\Psi_{A B}\right\rangle=\cos \theta\left|e_{A}, g_{B}\right\rangle+\sin \theta e^{i \phi}\left|g_{A}, e_{B}\right\rangle$ (with $0 \leq \theta \leq 2 \pi$ and $0 \leq \phi \leq \pi)$ as the initial state of atoms and assuming the initial cavity field to be in vacuum, ESD can occur for two types of initial atomic states. In order to study entanglement we will use the negativity $(N)$, which can be defined for two qubits as two times the modulus of the negative eigenvalue of the partial transposition of the state $\rho, \rho^{T_{A}}[25,26]$, if it exists. For short

$$
N(\rho)=2 \max \left\{0,-\lambda_{\min }\right\},
$$

where $\lambda_{\min }$ is the lowest eigenvalue of $\rho^{T_{A}}$. Our choice is motivated by the facts that the negativity is easy to calculate and provides full entanglement information for a two-qubit system. We also need to quantify three-party entanglement in the tree-qubit state $\left|\psi_{A B a}\right\rangle$, the three-tangle $\tau_{3}\left(\psi_{A B a}\right)$ has been introduced in Ref.[27]. It can be expressed by using the wave function coefficients $\left\{\psi_{000}, \psi_{001}, \cdots, \psi_{111}\right\}$ as

$$
\begin{aligned}
\tau_{3} & =4\left|d_{1}-2 d_{2}+4 d_{3}\right| \\
d_{1} & =\psi_{000}^{2} \psi_{111}^{2}+\psi_{001}^{2} \psi_{110}^{2}+\psi_{010}^{2} \psi_{101}^{2}+\psi_{100}^{2} \psi_{011}^{2} \\
d_{2} & =\psi_{000} \psi_{111} \psi_{011} \psi_{100}+\psi_{000} \psi_{111} \psi_{101} \psi_{010}+\psi_{000} \psi_{111} \psi_{110} \psi_{001} \\
& +\psi_{011} \psi_{100} \psi_{101} \psi_{010}+\psi_{011} \psi_{100} \psi_{110} \psi_{001}+\psi_{101} \psi_{010} \psi_{110} \psi_{001} \\
d_{3} & =\psi_{000} \psi_{110} \psi_{101} \psi_{011}+\psi_{111} \psi_{001} \psi_{010} \psi_{100}
\end{aligned}
$$

To figure out general conditions for the possible negativity of atomic ESD, we shall study two different atom-cavity models, which we refer to as Model 1 and Model 2. Model 1 is considered in section 2 , in this model one of two atoms is trapped in a single cavity, off-resonantly coupled to this cavity, and driven by a classic strong coherent field, while the other remains outside the cavity and has no environment. In section 3 we consider Model 2 which deals with a double driven JCM: each of the two strongly driven atoms interacts with its own cavity in the absence of any coupling between the atom-field subsystems. A generic result we have found out is that the atomic ESD always occurs in the conditions of the atom-field coupling larger than the atom-field detuning. Finally, we conclude in section 4.

\section{Model 1}

In this section we show a model in which a strongly driven atom $A$ is off-resonantly coupled to a single-mode cavity field $a$, while the other atom $B$ is isolated from all environment. The 
Hamiltonian of the system can be described by

$$
H=\frac{\hbar \omega_{a}}{2} \sigma_{A}^{z}+\frac{\hbar \omega_{b}}{2} \sigma_{B}^{z}+\hbar \omega_{f} a^{\dagger} a+\hbar \Omega\left(e^{-i \omega_{D} t} \sigma_{A}^{+}+e^{i \omega_{D} t} \sigma_{A}\right)+\hbar g\left(\sigma_{A}^{+} a+\sigma_{A} a^{\dagger}\right),
$$

where $\Omega$ is the Rabi frequency associated with the coherent driving field amplitude, $g$ is the atomcavity mode coupling constant, $a\left(a^{\dagger}\right)$ the field annihilation (creation) operator, $\sigma_{A}=\left|g_{A}\right\rangle\left\langle e_{A}\right|$ $\left(\sigma_{A}^{+}=\left|e_{A}\right\rangle\left\langle g_{A}\right|\right)$ the atomic lowering (raising) operator, and $\sigma_{A}^{z}=\left|e_{A}\right\rangle\left\langle e_{A}|-| g_{A}\right\rangle\left\langle g_{A}\right|$ the inversion operator. Considering the strong-driving regime for the interaction between the atom and the external coherent field $\Omega \gg\{g, \delta\}$ and making $\omega_{a}=\omega_{D}$, we can use the rotating-wave approximation (RWA) obtaining the effective Hamiltonian [28]

$$
H_{e f f}=\frac{\hbar g}{2}\left(\sigma_{A}^{+}+\sigma_{A}\right)\left(a e^{i \delta t}+a^{\dagger} e^{-i \delta t}\right) .
$$

where $\delta=\omega_{f}-\omega_{a}$ is the atom-cavity detuning.

We first choose the state $\left|\Psi_{A B}\right\rangle$ as an initial state of atoms $A$ and $B$, and assume the initial cavity field to be in vacuum ( $\mid$ Vacuum $\rangle$ means no photon in the single-mode cavity field). Thus the total system state at $t=0$ is

$$
\left.|\Psi(0)\rangle_{A B a}=\left(\cos \theta\left|e_{A}, g_{B}\right\rangle+\sin \theta e^{i \phi}\left|g_{A}, e_{B}\right\rangle\right) \otimes \mid \text { Vacuum }\right\rangle_{a}
$$

For simplicity, we make $\phi=0$. Then the evolved state in time $t$ will be $|\Psi(t)\rangle=U(t)|\Psi(0)\rangle$, and $U(t)=\exp \left(-i H_{e f f} t\right)$, so

$$
\begin{aligned}
|\Psi(t)\rangle_{A B a} & =\frac{\cos \theta+\sin \theta}{2}|\alpha\rangle\left|+_{A},+_{B}\right\rangle+\frac{\cos \theta-\sin \theta}{2}|\alpha\rangle\left|+_{A},-{ }_{B}\right\rangle \\
& -\frac{\cos \theta-\sin \theta}{2}|-\alpha\rangle\left|-_{A},+_{B}\right\rangle-\frac{\cos \theta+\sin \theta}{2}|-\alpha\rangle\left|-{ }_{A},-{ }_{B}\right\rangle,
\end{aligned}
$$

with $\alpha=\frac{g}{2 \delta}\left(1-e^{i \delta t}\right)$ and $\left| \pm_{X}\right\rangle=\left(\left|g_{X}\right\rangle \pm\left|e_{X}\right\rangle\right) / \sqrt{2}$, where $X=A, B$ and $\{|i\rangle\}_{i=1}^{4}=\left\{\mid{ }_{A}\right.$ $\left.\left.+_{B}\right\rangle,\left|+_{A}-{ }_{B}\right\rangle,\left|-{ }_{A}+_{B}\right\rangle,\left|-{ }_{A}-{ }_{B}\right\rangle\right\}$ is the rotated basis of the atomic Hilbert space. We now try to estimate the atomic entanglement of the state Equation(6), and we notice that the cavity field in the general nonorthogonal coherent state $|\alpha\rangle$ and $|-\alpha\rangle$. So we can define $|0\rangle=|\alpha\rangle$, $|1\rangle=(|-\alpha\rangle-P|\alpha\rangle) / \sqrt{1-P^{2}}$ with $P=\exp \left(\frac{g^{2}}{\delta^{2}}(\cos \delta t-1)\right)$ for the cavity field subsystem. Then Equation(6) can be written as

$$
\begin{aligned}
|\Psi(t)\rangle & =\left(\frac{\cos \theta+\sin \theta}{2}\left|+{ }_{A},+_{B}\right\rangle+\frac{\cos \theta-\sin \theta}{2}\left|{ }_{A},-_{B}\right\rangle\right. \\
& \left.-\frac{\cos \theta-\sin \theta}{2} P\left|-{ }_{A}+{ }_{B}\right\rangle-\frac{\cos \theta+\sin \theta}{2} P\left|-{ }_{A}-{ }_{B}\right\rangle\right)|0\rangle \\
& -\left(\frac{\cos \theta-\sin \theta}{2} \sqrt{1-P^{2}}\left|-{ }_{A}+{ }_{B}\right\rangle+\frac{\cos \theta+\sin \theta}{2} \sqrt{1-P^{2}}\left|-{ }_{A}-{ }_{B}\right\rangle\right)|1\rangle
\end{aligned}
$$




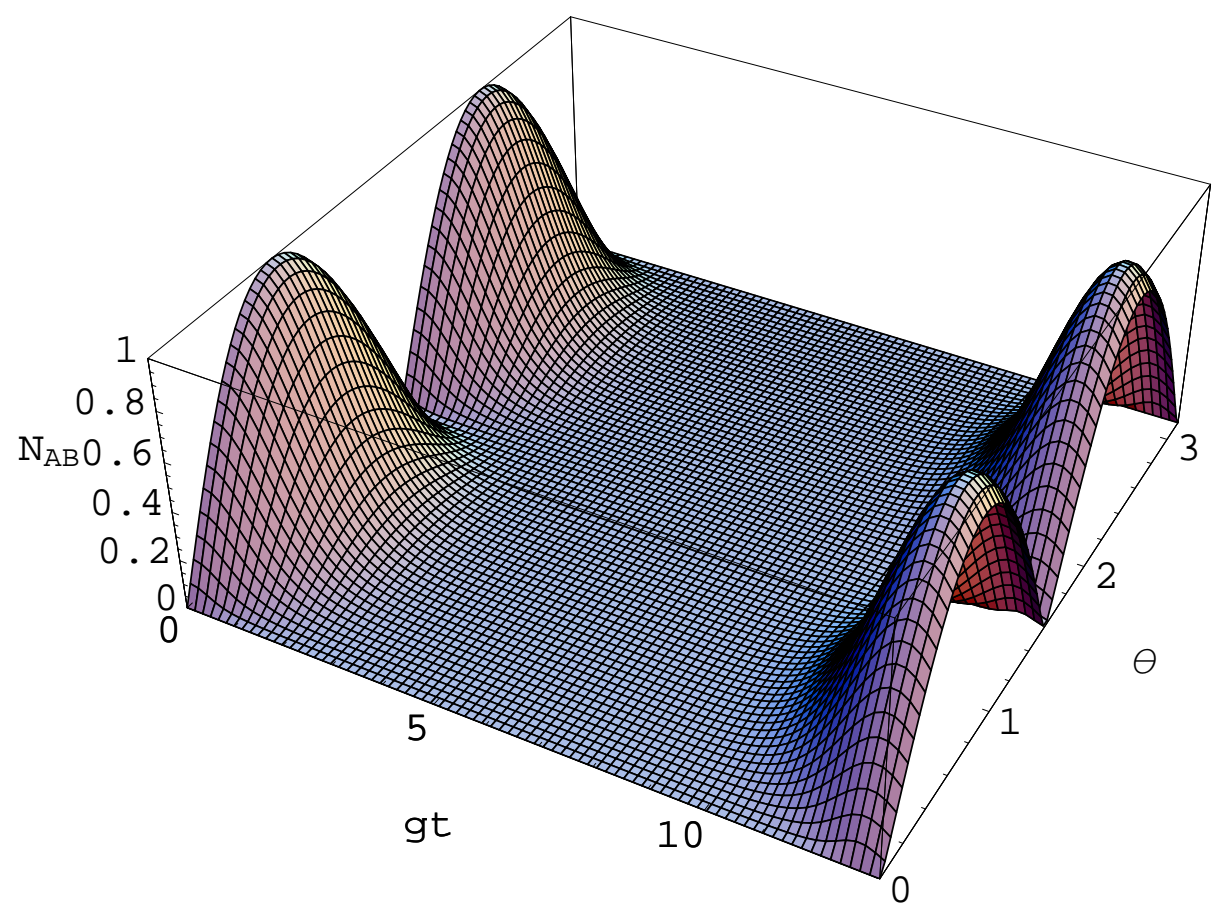

FIG. 1: $N\left(\rho_{A B}^{\Psi}(t)\right)$ as a function of $\theta$ and the dimensionless time $g t$ for $g / \delta=2$, with $\delta=0.5 g$ in Model 1.

In order to calculate the negativity of atoms, we trace out the mode $a$, the expression for $\rho_{A B}^{\Psi}(t)$ in the rotated basis of the atomic Hilbert space

$$
\begin{aligned}
& \rho_{11}=\rho_{44}=\left(\frac{\cos \theta+\sin \theta}{2}\right)^{2}, \rho_{22}=\rho_{33}=\left(\frac{\cos \theta-\sin \theta}{2}\right)^{2}, \\
& \rho_{14}=\rho_{41}=-\left(\frac{\cos \theta+\sin \theta}{2}\right)^{2} P, \rho_{23}=\rho_{32}=-\left(\frac{\cos \theta-\sin \theta}{2}\right)^{2} P, \\
& \rho_{12}=\rho_{21}=\rho_{34}=\rho_{43}=\frac{\cos ^{2} \theta-\sin ^{2} \theta}{4}, \\
& \rho_{13}=\rho_{31}=\rho_{42}=\rho_{24}=-\frac{\cos ^{2} \theta-\sin ^{2} \theta}{4} P .
\end{aligned}
$$

The negativity of $\rho_{A B}^{\Psi}(t)$ is

$$
N\left(\rho_{A B}^{\Psi}(t)\right)=2 \max \left\{0,-\frac{1}{4}\left(1-P-\sqrt{1+P^{2}-2 P \cos 4 \theta}\right)\right\} .
$$

The negativity $N\left(\rho_{A B}^{\Psi}(t)\right)$ has been calculated, as shown in Fig.1 and Fig.2, for the different $g / \delta$. Obviously the negativity is fluctuating with dimensionless time $g t$ and the mixing angle $\theta$, and it can be zero in a finite amount of time (the so-called entanglement sudden death). An important point is that ESD is sensitive to the value of $g / \delta$ as displayed in Fig.1 and Fig.2. For $g / \delta>1$, 


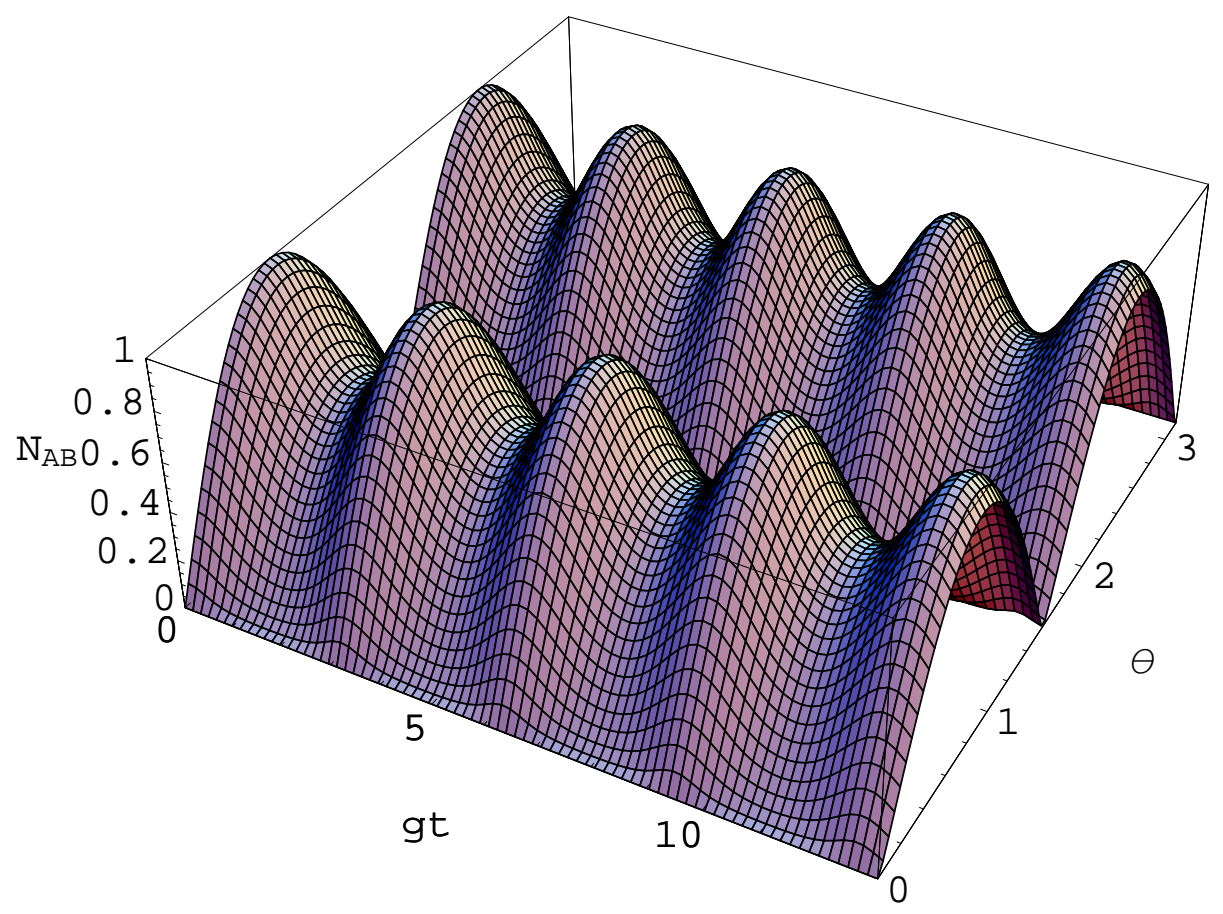

FIG. 2: $N\left(\rho_{A B}^{\Psi}(t)\right)$ as a function of $\theta$ and the dimensionless time $g t$ for $g / \delta=0.5$, with $\delta=2 g$ in Model 1.

ESD happens readily (Fig.1), and the larger the value of $g / \delta$ is, the more easily the ESD appears. When $g / \delta \leq 1$, ESD will not appear at any time (Fig.2). This phenomenon shows that ESD is related to $g / \delta$ completely in this model. Moreover, As we study the entanglement dynamics under a close system, Fig.1 also shows that sudden death and resurrection of atomic entanglement can occur periodically as well as alternately. After entanglement of two atoms occurs sudden death for a finite time, atomic entanglement can revive again gradually. We have verified that when the ratio $g / \delta$ is larger than the one used in Fig.1, the region of ESD and entanglement revivals increases, and they last for a longer time. On the other hand, when we decrease $g / \delta$, the ESD regions shrink and the period of the entanglement oscillations becomes smaller.

The character revealed by Fig. 3 is that $\delta$ influences the period of the ESD and $g$ is related to the velocity of the $A B$ subsystem's disentanglement. If we want to acquire a long-time $\mathrm{ESD}$, then we can choose a smaller atom-field detuning $\delta($ Fig.3( $a)$ ). While $\delta$ is a constant, the $A B$ subsystem can rapidly disentangle with the larger $g$ (Fig.3(b)). A physical interpretation of the result is that the more the atom to the cavity mode couples, the easier the initial entanglement decays. Then we consider the atom and the cavity under resonance condition $(\delta=0)$ or the large detuning regime 

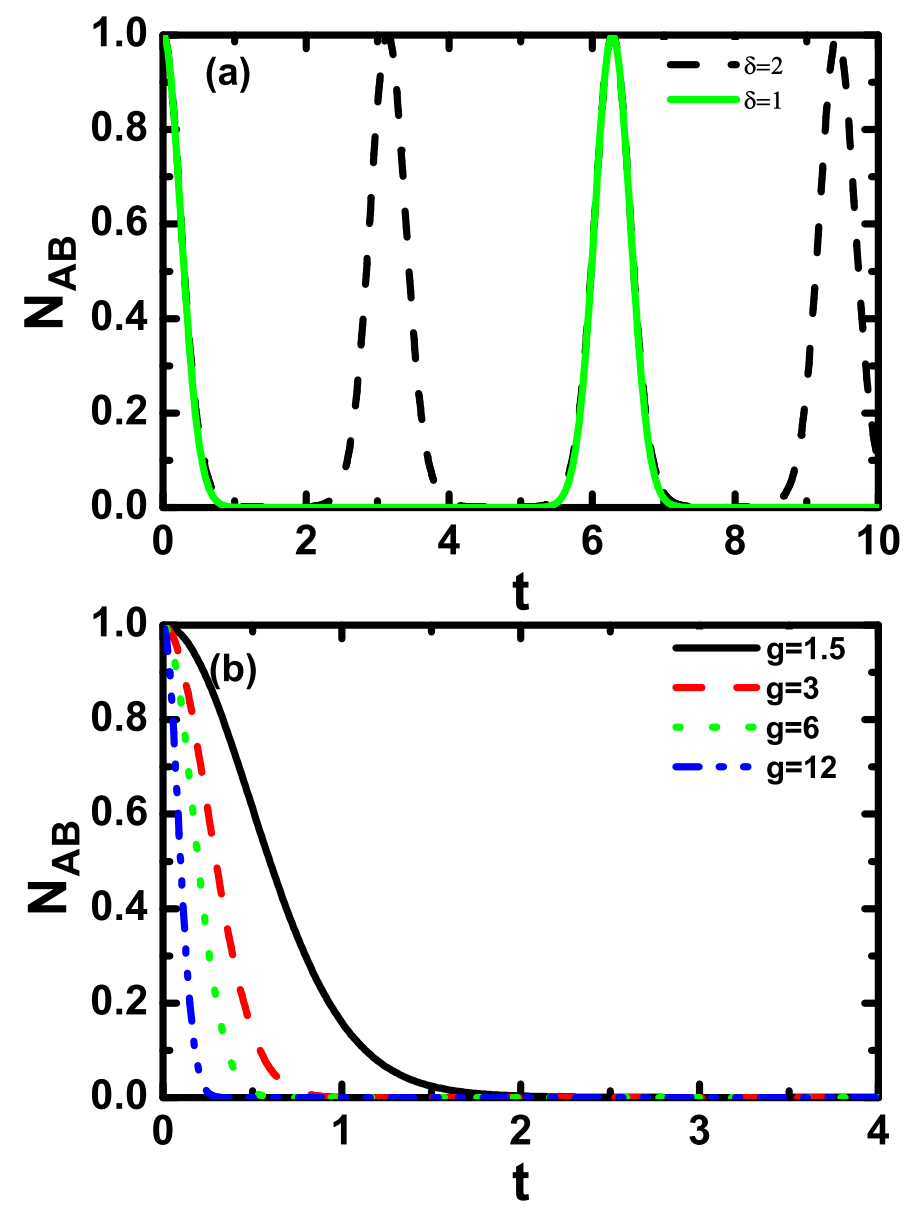

FIG. 3: (a) $N\left(\rho_{A B}^{\Psi}(t)\right)$ as a function of time $t$ for $g=4, \theta=\pi / 4$ in Model 1 , (b) $N\left(\rho_{A B}^{\Psi}(t)\right)$ as a function of time $t$ for $\delta=1, \theta=\pi / 4$ in Model 1 .

$(\delta \gg g)$. Firstly, in the resonant regime the negativity of $\rho_{A B}^{\Psi}(t)$ decreases at the beginning, and then vanishes at any time and resurrection of atomic entanglement can not happen forever under this condition(Fig.4), so the strongly driven model under the resonant regime can be considered as an information eraser. Secondly, entangled states are generally very fragile against interaction with environments, but in our present scheme, the energy exchange between atom and cavity do not exist under the atom-cavity large detuning regime, so the initial entanglement of atoms can be preserved during system evolution, which is shown as Fig.5. That is to say, the strongly driven and large detuning model can be used to preserve the information (entanglement) in quantum processing and quantum computing. 


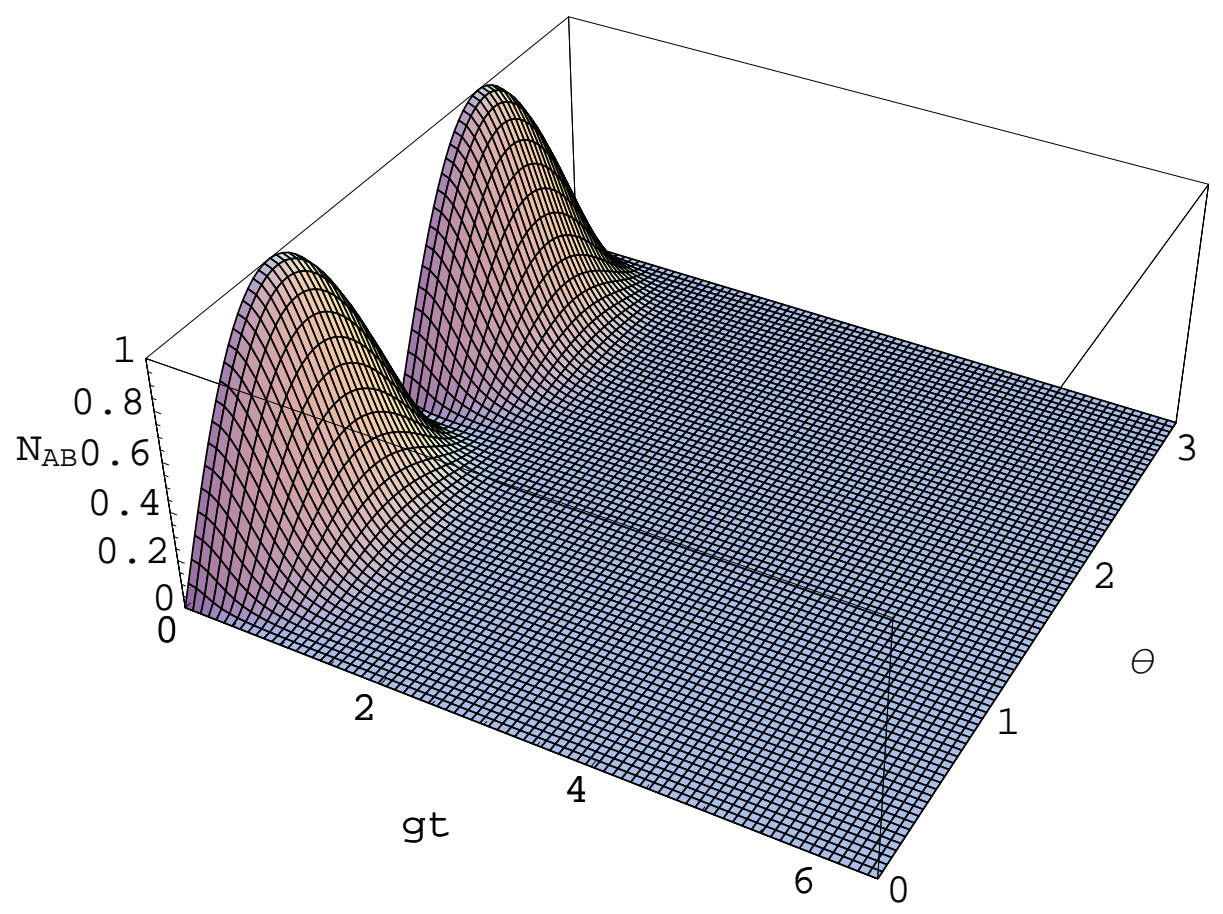

FIG. 4: $N\left(\rho_{A B}^{\Psi}(t)\right)$ as a function of $\theta$ and the dimensionless time $g t$ for $\delta=0$ in Model 1

Now we move to the negativity $N\left(\rho_{A a}^{\Psi}(t)\right)$ and $N\left(\rho_{B a}^{\Psi}(t)\right)$ and the three-tangle $\tau\left(\rho_{A B a}^{\Psi}(t)\right)$, and we acquire the following

$$
\begin{gathered}
N\left(\rho_{A a}^{\Psi}(t)\right)=2 \max \left\{0, Q_{A a}\right\}, \\
N\left(\rho_{B a}^{\Psi}(t)\right)=2 \max \left\{0, Q_{B a}\right\}, \\
\tau\left(\rho_{A B a}^{\Psi}(t)\right)=\frac{1}{2}\left(1-P^{2}\right)(1-\cos 4 \theta),
\end{gathered}
$$

if $0<\cos 2 \theta<1$, then $Q_{A a}=-\frac{1}{8}\left(2-2 \cos 2 \theta-\sqrt{2} \sqrt{3+\left(4-8 P^{2}\right) \cos 2 \theta+\cos 4 \theta}\right)$ and $Q_{B a}=-\frac{1}{2}(1-P \cos 2 \theta)$, and if $-1<\cos 2 \theta<0$, then $Q_{A a}=-\frac{1}{8}(2+2 \cos 2 \theta-$ $\left.\sqrt{2} \sqrt{3+\left(8 P^{2}-4\right) \cos 2 \theta+\cos 4 \theta}\right)$ and $Q_{B a}=-\frac{1}{2}(1+P \cos 2 \theta)$. Due to $0<P<1$, then $Q_{B a}<0$, the negativity $N\left(\rho_{B a}^{\Psi}(t)\right)=0$. In the strongly driven regime, $B$ is isolated from all environment, and the strong classical field that drives the atom $A$ can be used to enhance the atom $(A)$-cavity field $(a)$ interaction. The entanglement between $A$ and cavity $a$ can appear during the system evolution, but $B$ and cavity $a$ can not entangle with each other in the strongly driven regime(that is different from the standard J-C Model [21]). It is interesting to find that while the $A B$ subsystem appears the long-time ESD, the entanglement of the $A a$ subsystem and the threetangle of the whole system are all on the long-time invariable entanglement(as shown in Fig.6(a)). 


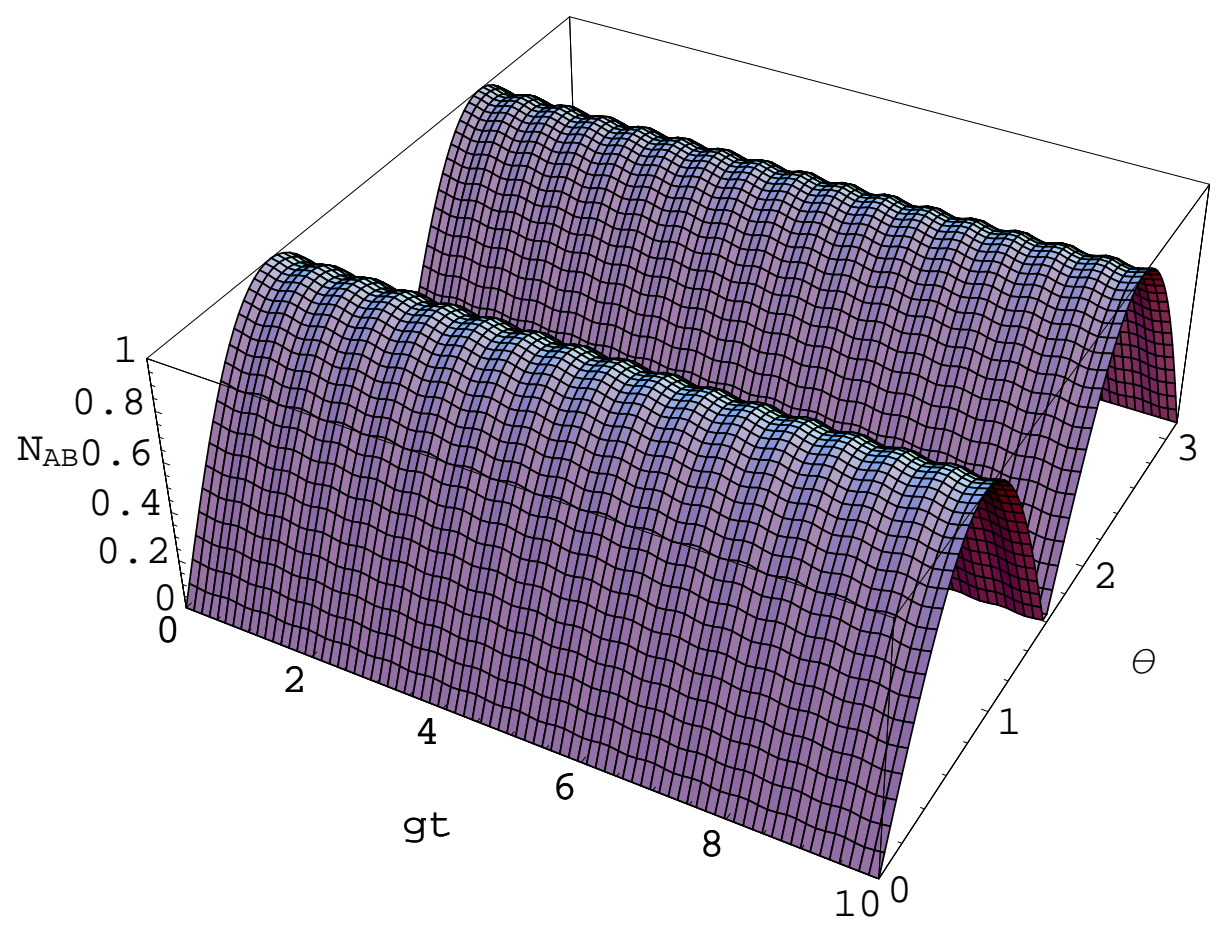

FIG. 5: $N\left(\rho_{A B}^{\Psi}(t)\right)$ as a function of $\theta$ and the dimensionless time $g t$ for $\delta=10 g$, in the large detuning regime in Model 1.

It is clearly shown that the three-tangle $\tau_{A B a}$ can be influenced by the initial state $\left|\Psi_{A B}(0)\right\rangle$ in Fig.6(b). On the condition $\theta=\pi / 4$ (the initial state is on the maximum entanglement), when $A B$ subsystem's entanglement vanishes for a long time, the three-tangle of the whole system can achieve the maximum value 1 at the same time and the entanglement between $A$ and $a$ can not appear for any time. That is to say, the $A B$ subsystem's entanglement is transferred to the whole system's entanglement thoroughly (Fig.6(c)). So, we can acquire the three-partite system's longtime maximum entanglement in the strongly driven regime (In Model 1).

Next, we choose $\left|\Phi_{A B}\right\rangle$ to be the initial atomic state. Then the initial total system state is

$$
\left.\Phi_{A B a}(0)=\left(\cos \theta\left|e_{A}, e_{B}\right\rangle+\sin \theta e^{i \phi}\left|g_{A}, g_{B}\right\rangle\right) \otimes \mid \text { Vacuum }\right\rangle_{a},
$$



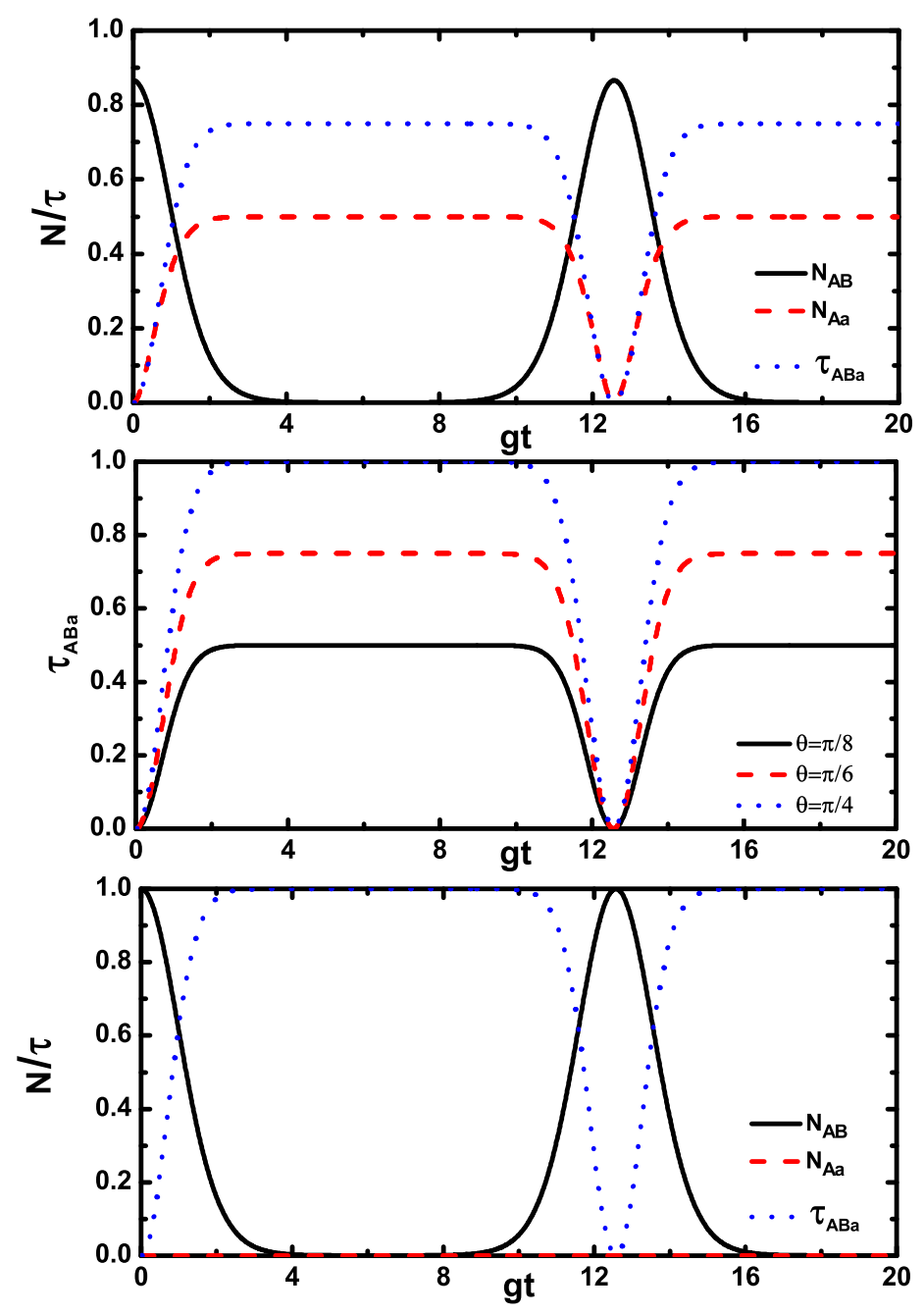

FIG. 6: $N\left(\rho_{A B}^{\Psi}(t)\right) / N\left(\rho_{A a}^{\Psi}(t)\right) / \tau\left(\rho_{A B a}^{\Psi}(t)\right)$ as a function of the dimensionless time $g t$ in Model 1 , (a) $\delta=$ $0.5 g, \theta=\frac{\pi}{6},(\mathrm{~b}) \delta=0.5 g$, (c) $\delta=0.5 g, \theta=\frac{\pi}{4}$.

with $\phi=0$, and for $\rho_{A B a}^{\Phi}(t)$ we have the expressions as follows

$$
\begin{aligned}
& \rho_{11}=\rho_{44}=\left(\frac{\cos \theta+\sin \theta}{2}\right)^{2}, \rho_{22}=\rho_{33}=\left(\frac{\cos \theta-\sin \theta}{2}\right)^{2}, \\
& \rho_{14}=\rho_{41}=\left(\frac{\cos \theta+\sin \theta}{2}\right)^{2} P, \rho_{23}=\rho_{32}=\left(\frac{\cos \theta-\sin \theta}{2}\right)^{2} P, \\
& \rho_{12}=\rho_{21}=\rho_{34}=\rho_{43}=-\frac{\cos ^{2} \theta-\sin ^{2} \theta}{4}, \\
& \rho_{13}=\rho_{31}=\rho_{42}=\rho_{24}=-\frac{\cos ^{2} \theta-\sin ^{2} \theta}{4} P .
\end{aligned}
$$


The negativity of $\rho_{A B}^{\Phi}(t)$ is satisfied with

$$
N\left(\rho_{A B}^{\Phi}(t)\right)=N\left(\rho_{A B}^{\Psi}(t)\right) .
$$

Equation(15) reveals that the behavior of $N\left(\rho_{A B}^{\Phi}(t)\right)$ dependent on $g / \delta$ is equal to that of $N\left(\rho_{A B}^{\Psi}(t)\right)$. We also calculate the negativity $N\left(\rho_{A a}^{\Phi}(t)\right)$ and $N\left(\rho_{B a}^{\Phi}(t)\right)$ and the three-tangle $\tau\left(\rho_{A B a}^{\Phi}(t)\right)$, and find that they are all equal to $N\left(\rho_{A a}^{\Psi}(t)\right), N\left(\rho_{B a}^{\Psi}(t)\right)$ and $\tau\left(\rho_{A B a}^{\Psi}(t)\right)$.

Consequently, for Model 1, where only one driven atom interacts with its environment, if the atom is not driven and the cavity field is in the vacuum initially, ESD does not occur either for $\rho_{A B}^{\Phi}(0)$ or $\rho_{A B}^{\Psi}(0)$. In contrast, however, if the atom is driven by a strong classical field and both the atom-cavity coupling and the atom-cavity detuning satisfy $g / \delta>1$, the atomic subsystem $A B$ always evolves via ESD, independent of the type of initial atomic state(i.e. it never matters if it is $\rho_{A B}^{\Phi}(0)$ or $\left.\rho_{A B}^{\Psi}(0)\right)$.

\section{Model 2}

The double JCM, which has been intensively investigated recently [19], has shown that if atoms $A$ and $B$ are prepared in the $\left|\Phi_{A B}\right\rangle$-type Bell-like pure state initially, then the atomic entanglement dies in a finite time and remains dead for some time before reviving itself again, i.e., ESD occurs, whereas the $\left|\Psi_{A B}\right\rangle$-type Bell-like pure state dose not exhibit ESD at all. However, these investigations are confined to the atom-field under full resonance conditions. Our purpose here is focus on the differences that may appear on the conditions the atoms $A$ and $B$ independently driven by a strongly external classical field and the atom-cavity off resonance.

In Model 2, we consider two remote two-level atoms $A$ and $B$ which are first prepared to be in an entangled state, and then let each atom couple with a single-mode cavity respectively. During the interaction with the single-mode cavity, the two atoms are independently driven by a strongly classical field. In the strong-driving regime, The effective Hamiltonian governing Model 2 is of the form

$$
H_{e f f}=\sum_{k=A, B} H_{e f f}^{k}
$$

where

$$
H_{e f f}^{k}=\frac{\hbar g_{k}}{2}\left(\sigma_{k}^{+}+\sigma_{k}\right)\left(a_{k} e^{i \delta_{k} t}+a_{k}^{\dagger} e^{-i \delta_{k} t}\right) .
$$

The total system state at $t=0$ of the form

$$
\left.\left.|\Psi(0)\rangle_{A B a b}=\left(\cos \theta\left|e_{A}, g_{B}\right\rangle+\sin \theta e^{i \phi}\left|g_{A}, e_{B}\right\rangle\right) \otimes \mid \text { Vacuum }\right\rangle_{a} \mid \text { Vacuum }\right\rangle_{b}
$$


also with $\phi=0$, then the evolved state in time $t$ will be

$$
\begin{aligned}
|\Psi(t)\rangle_{A B a b} & =\exp \left(-i H_{e f f} t\right)|\Psi(0)\rangle_{A B a b} \\
& =\frac{\cos \theta+\sin \theta}{2}\left|\alpha_{a}, \beta_{b}\right\rangle\left|+_{A},+_{B}\right\rangle+\frac{\cos \theta-\sin \theta}{2}\left|\alpha_{a},-\beta_{b}\right\rangle\left|+_{A},-_{B}\right\rangle \\
& -\frac{\cos \theta-\sin \theta}{2}\left|-\alpha_{a}, \beta_{b}\right\rangle\left|-_{A},+_{B}\right\rangle-\frac{\cos \theta+\sin \theta}{2}\left|-\alpha_{a},-\beta_{b}\right\rangle\left|-{ }_{A},-{ }_{B}\right\rangle,
\end{aligned}
$$

where $\{|i\rangle\}_{i=1}^{4}=\left\{\left|+_{A}+_{B}\right\rangle,\left|+_{A}-_{B}\right\rangle,\left|-_{A}+{ }_{B}\right\rangle,\left|-_{A}-_{B}\right\rangle\right\}$ is the rotated basis of the atomic Hilbert space. Similar to Equation(6), we also define

$$
\begin{aligned}
& \left|0_{a}\right\rangle=\left|\alpha_{a}\right\rangle,\left|1_{a}\right\rangle=\left(\left|-\alpha_{a}\right\rangle-P_{A}\left|\alpha_{a}\right\rangle\right) / \sqrt{1-P_{A}^{2}} \\
& \left|0_{b}\right\rangle=\left|-\beta_{b}\right\rangle,\left|1_{b}\right\rangle=\left(\left|\beta_{b}\right\rangle-P_{B}\left|-\beta_{b}\right\rangle\right) / \sqrt{1-P_{B}^{2}}
\end{aligned}
$$

where $P_{A}=\exp \left(\frac{g_{A}^{2}}{\delta_{A}^{2}}\left(\cos \delta_{A} t-1\right)\right), P_{B}=\exp \left(\frac{g_{B}^{2}}{\delta_{B}^{2}}\left(\cos \delta_{B} t-1\right)\right)$. The reduced density matrix $\rho_{A B}^{\Psi}(t)$ is

$$
\begin{aligned}
& \rho_{11}=\rho_{44}=\left(\frac{\cos \theta+\sin \theta}{2}\right)^{2}, \rho_{22}=\rho_{33}=\left(\frac{\cos \theta-\sin \theta}{2}\right)^{2}, \\
& \rho_{14}=\rho_{41}=-\left(\frac{\cos \theta+\sin \theta}{2}\right)^{2} P_{A} P_{B}, \rho_{23}=\rho_{32}=-\left(\frac{\cos \theta-\sin \theta}{2}\right)^{2} P_{A} P_{B}, \\
& \rho_{12}=\rho_{21}=\rho_{34}=\rho_{43}=\frac{\cos ^{2} \theta-\sin ^{2} \theta}{4} P_{B}, \\
& \rho_{13}=\rho_{31}=\rho_{42}=\rho_{24}=-\frac{\cos ^{2} \theta-\sin ^{2} \theta}{4} P_{A} .
\end{aligned}
$$

The negativity of $\rho_{A B}^{\Psi}(t)$ is

$N\left(\rho_{A B}^{\Psi}(t)\right)=2 \max \left\{0,-\frac{1}{8}\left(2-2 P_{A} P_{B}-\sqrt{2} \sqrt{\left(1+P_{A}^{2}\right)\left(1+P_{B}^{2}\right)+\left(P_{A}^{2}+P_{B}^{2}-1-4 P_{A} P_{B}-P_{A}^{2} P_{B}^{2}\right) \cos 4 \theta}\right)\right\}$

The negativity of $\rho_{A B}^{\Psi}(t)$ is given by Equation(23) in full similarity with Model 1, ESD always occurs for $g_{A} / \delta_{A}>1$ and $g_{B} / \delta_{B}>1$. The physical interpretation of the result is that $g_{A} / \delta_{A}>1$ (or $g_{B} / \delta_{B}>1$ ) means the strong coupling regime. Because the atom $A$ (or $B$ ) and cavity $a$ (or $b$ ) couple with each other strongly, the entanglement of the $A B$ subsystem can occur ESD phenomenon. $g_{A}$ and $g_{B}$ are related to the velocity of the initial state's disentanglement, and $\delta_{A}$, $\delta_{B}$ influence the ESD's period. In addition, the ESD also appears on the condition $g_{A} / \delta_{A} \geq 1$, $\delta_{B} \geq g_{B}$ or $g_{B} / \delta_{B} \geq 1, \delta_{A} \geq g_{A}$, even when $\delta_{A}>g_{A}, \delta_{B}>g_{B}$ (but when $\delta_{A}>g_{A}$ or $\delta_{B}>g_{B}, \delta_{A}$ and $\delta_{B}$ do not correspond to the large detuning regime), and is shown as Fig.7 and Fig.8, in which the ESD phenomenon can also occur in the case when $\delta_{A}$ and $\delta_{B}$ are larger than $g_{A}$ and $g_{B}$ (but not 


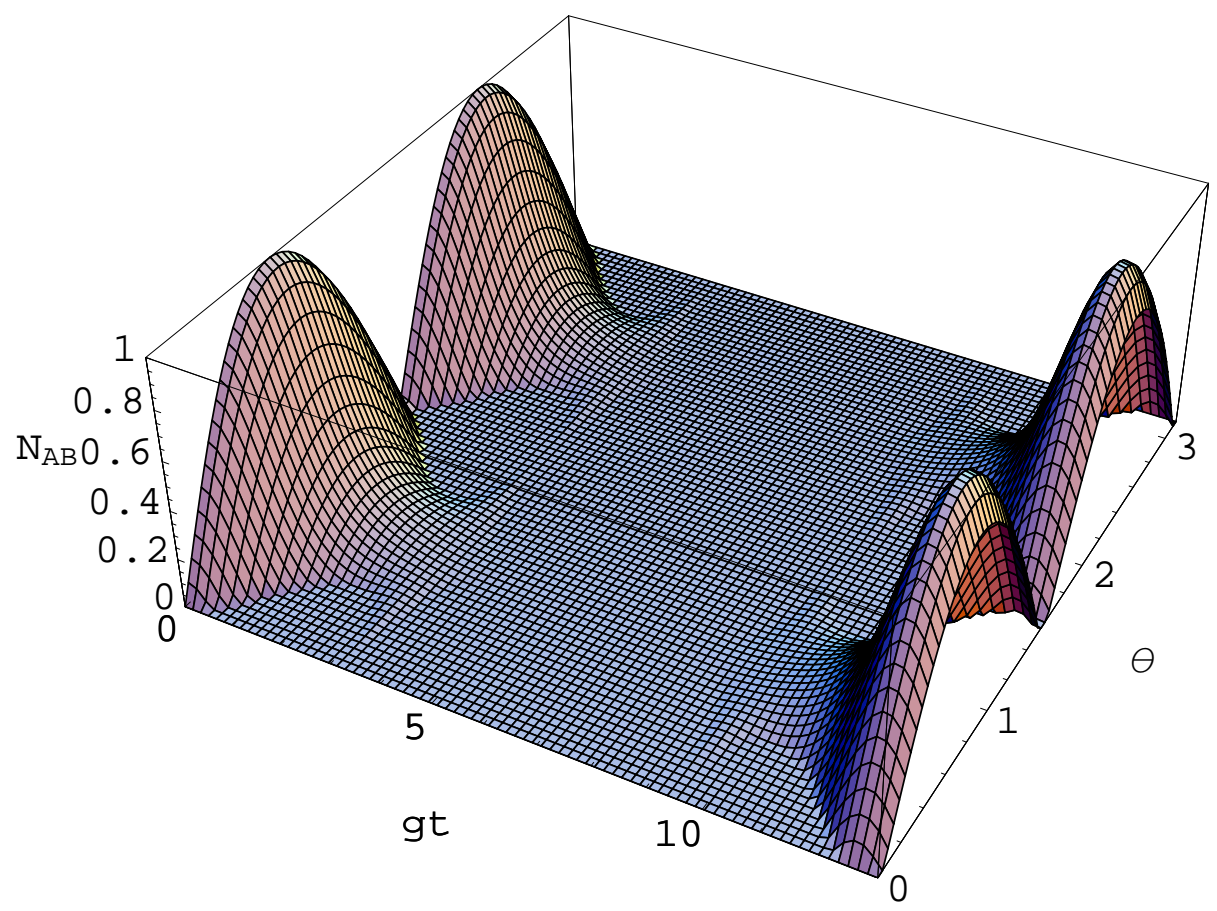

FIG. 7: $N\left(\rho_{A B}^{\Psi}(t)\right)$ as a function of $\theta$ and the dimensionless time $g t$ for $\delta_{A}=0.5 g, \delta_{B}=2 g$ in Model 2.

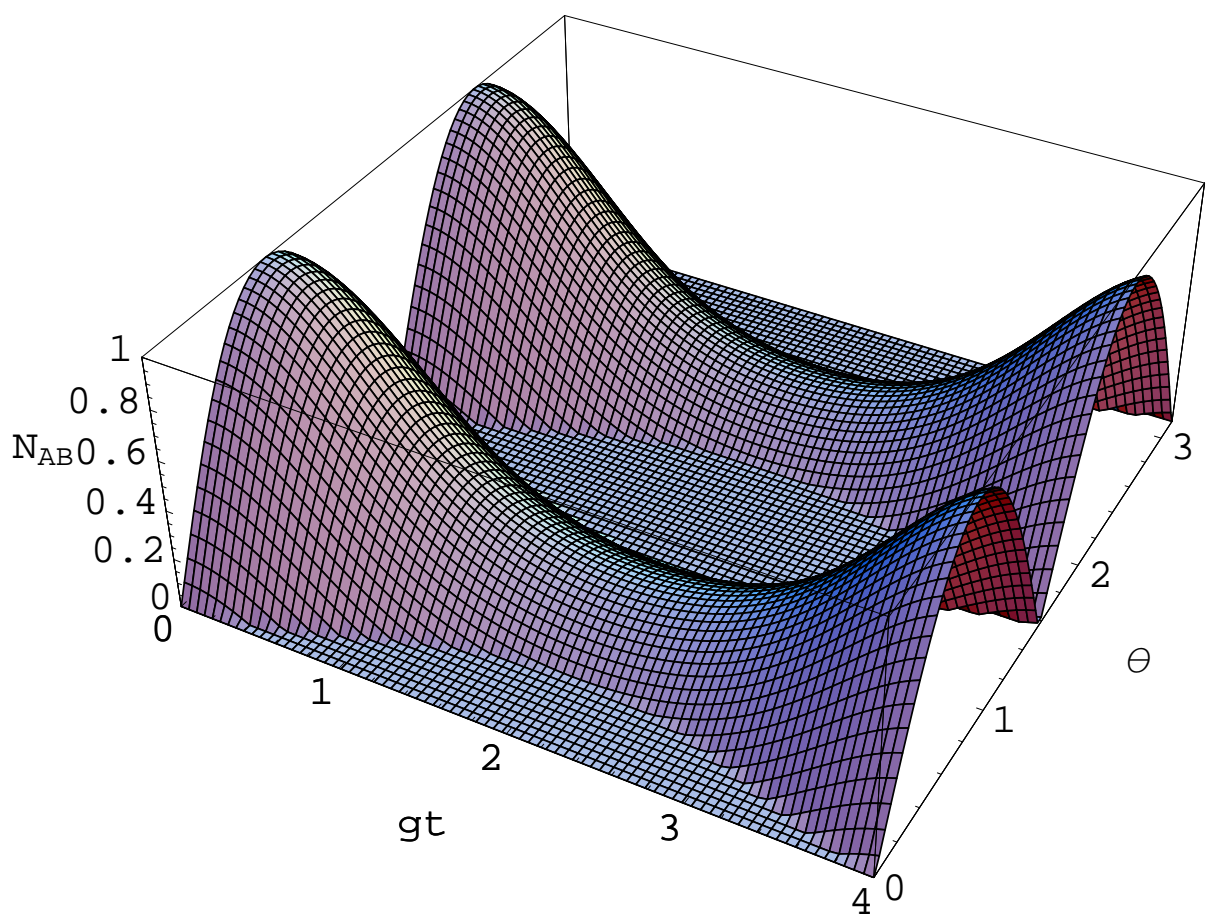

FIG. 8: $N\left(\rho_{A B}^{\Psi}(t)\right)$ as a function of $\theta$ and the dimensionless time $g t$ for $\delta_{A}=\delta_{B}=1.5 g$ in Model 2 . 
by too much). As we all know, the larger atom-cavity detuning means that the interaction between the atom and cavity becomes small, for example, in the large detuning regime, the cavity and the atom can not exist energy exchange. So when $\delta_{A}$ and $\delta_{B}$ are much larger than $g_{A}$ and $g_{B}$, the ESD will disappear. If we consider the driven atoms and the cavities under resonance condition $\left(\delta_{A}=\delta_{B}=0\right)$, the negativity of $\rho_{A B}^{\Psi}(t)$ decreases at the beginning, and then vanishes for all time (the same as Fig.4). So Model 2 under the resonance regime can also be used as an information eraser.

For another type of atomic initial state $\left|\Phi_{A B}\right\rangle$, it can be verified that the negativity $N\left(\rho_{A B}^{\Phi}(t)\right)$ is also equal to $N\left(\rho_{A B}^{\Psi}(t)\right)$. Through calculating the negativity $N_{A a}, N_{B b}, N_{A b}$ and $N_{B a}$ in Model 2, we find that the entanglement of $A a$ and $B b$ can occur during the system evolution and can be on a long-time invariable entanglement when the $A B$ subsystem is occurring the ESD. Because of no existing interaction in the $B a$ subsystem (or $A b$ subsystem), it is impossible to entangle $A b$ (or $\mathrm{Ba}$ ) in the strongly driven regime (these results are not similar to the standard double J-C Model [19]). Hence, our conclusion regarding Model 2 is that the driven atomic subsystem $A B$ always suffers ESD if the atom-cavity coupling and the atom-detuning are satisfied with the condition $g_{A} / \delta_{A}>1, g_{B} / \delta_{B}>1$ or $g_{A} / \delta_{A} \geq 1, \delta_{B} \geq g_{B}$ or $g_{B} / \delta_{B} \geq 1, \delta_{A} \geq g_{A}$, even when $\delta_{A}>g_{A}, \delta_{B}>g_{B}$ (but $\delta_{A}$ and $\delta_{B}$ can not correspond to the large detuning regime), independent of the type of the atomic initial state which may be either $\rho_{A B}^{\Phi}(0)$ or $\rho_{A B}^{\Psi}(0)$.

\section{Conclusion}

We have described the entanglement evolution of two two-level atoms off-resonantly coupled to cavity fields. In Model 1 , one of two atoms is trapped in a single cavity, off-resonantly coupled to this cavity, and driven by a classic strong coherent field, while the other remains outside the cavity and has no environment. However, in Model 2, each of the two strongly driven atoms interacts with its own cavity in the absence of any coupling between the atom-field subsystems. There are different available or forthcoming routes to the implementation of our model. In the microwave regime of cavity QED, pairs of atoms excited to Rydberg levels cross a high-Q superconductive cavity with negligible spontaneous emission during the interaction [29]. In the optical regime the application of cooling and trapping techniques in cavity QED [23] allows the deterministic loading of single atoms in a high-finesse cavity, with accurate position control and trapping times of many seconds [24]. In this regime laser-assisted three-level atoms can behave as effective 
two-level atoms [30]. On the other hand, trapped atomic ions can remain in an optical cavity for an indefinite time in a fixed position, where they can couple to a single mode without coupling rate fluctuations [31]. These systems are quite promising for our purposes and could become almost ideal in the case of achievement of the strong coupling regime.

Under off-resonance conditions and starting from the vacuum state of the cavity fields, for negligible atomic decays and cavity leakage, we solved exactly the system dynamics for two types of initial preparation of the atom pairs $\left(\left|\Phi_{A B}\right\rangle\right.$ and $\left.\left|\Psi_{A B}\right\rangle\right)$. Thus we found conditions for the negativity of the so-called atomic ESD. Namely, the initial entanglement of atoms, if any, will eventually suffer a sudden death, if the atom-cavity coupling and the atom-cavity detuning are satisfied with $g / \delta>1$ (in Model 1 ), while in Model 2 the system is accordance to $g_{A} / \delta_{A} \geq 1, g_{B} / \delta_{B} \geq 1$ or $g_{A} / \delta_{A}>1, \delta_{B}>g_{B}$ and/or $g_{B} / \delta_{B}>1, \delta_{A}>g_{A}$ (but $\delta_{A}$ and $\delta_{B}$ can not correspond to the large detuning regime). Furthermore, it is interesting to note that such conditions for ESD do not depend on the concrete type of the initial state of atoms. The atom-cavity detuning $\delta$ influences the period of ESD and the atom-cavity coupling $g$ is related to the velocity of the $A B$ subsystem's disentanglement.

In the atom-cavity resonance regime, the negativity of the atomic subsystem decreases at the beginning, and then vanishes for any time, so the strongly driven model (such as Model 1 and Model 2) under the resonance regime can be used as an information eraser. Entangled states are generally very fragile against interaction with environments, but in our present scheme, the energy exchange between atoms and cavities do not exist under the atom-cavity large detuning regime, so the initial entanglement of atoms is preserved during system evolution. That is to say, the strongly driven and large detuning model can be used to preserve the information (entanglement) in quantum information processing and quantum computing.

In the strongly driven regime, we note the negativity of two atoms is a periodic function of time in the close system. It means that atoms evolve quasiperiodically between the entangled and disentangled states. This result is different from refs.[15, 16], ref.[15] shows that the negativity of the bipartite system is a mononically decreasing function of time when the first qubit is trapped in a infinite heat bath and the second qubit has no environment. But authors in ref.[15] also have proposed that in the case when the second qubit is set in a finite and controlling quantum environment, the dynamics of entanglement can oscillate, this nonmontonic behavior appears to be linked to the non-markovian character of the dynamics. That is to say, non-markovian entanglement can evolve quasiperiodically between the entangled and disentangled states in the open systems. From 
the paper [15] we acquire the degree of entanglement at certain time intervals can be manipulated by means of an appropriate choice of the initial state of the controlling quantum environment. Our results in the strongly driven regime manifest the entanglement of two atoms can be manipulated through controlling the atom-cavity detuning and atom-cavity coupling.

\section{Acknowledgments}

YJZ, ZXM and YJX are supported by the National Science Foundation of China under Grant No.10774088, the Key Program of National Science Foundation of China under Grant No.10534030.

\section{References}

[1]Nielsen M A and Chuang I L Quantum Computation and Quantum Information(Cambridge University Press, New York, 2000).

[2]Yu T and Eberly H J 2004 Phys. Rev. Lett. 93140404.

[3]Jakobczyk L and Jamroz A, 2004 Phys. Lett. A 33335.

[4]Bandyopadhyay S and Lidar D A 2004 Phys. Rev. A 70 010301(R).

[5]Yu T and Eberly H J 2006 Phys. Rev. A 97140403.

[6]Dodd P J and Halliwell J J, 2004 Phys. Rev. A 69052105.

[7]Ban M , 2006 J. Phys. A. 391927.

[8]Solenov D, Tolkunov D and Privman 2006 Phys. Lett. 35981.

[9]Zyczkowski K, Horodecki P Horodecki M and Horodecki R 2002 Phys. Rev. A 65012101.

[10]Diosi L in Irreversible Quantum Dynamics edited by Benati F and Floreanini (Spring, New York, 2003) pp.157-163.

[11]López C E, Romero G, Lastra F, Solano E and Retamal J C 2008 Phys. Rev. Lett. 101080503 ;

[12]Carvalho A R R, Mintert F and Buchleitner A 2007 Euro. Phys. J. D 41425.

[13]Derkacz L and Jakobczyk L 2006 Phys. Rev. A 74032313.

[14]Gordon G and Kurizki G 2006 Phys. Rev. Lett. 97110503. 
Carvalho A R R, Mintert F and Buchleitner A 2004 Phys. Rev. Lett. 93230501.

[15]Dajka J, Mierzejewski M and Luczka J 2008 Phys. Rev. A. 77042316.

[16]Dajka J and Luczka J 2008 Phys. Rev. A. 77062303.

[17]Santos M F, Milman P and Davidovich et al 2006 Phys. Rev. A. 73022313.

[18]Walborn S P, Ribeiro P H S, Davidovich L, Mintert F and Bchleitner A 2006 Nature 4401022.

[19]Yönaç M, Yu T and Eberly J H 2007 J. Phys.B: At. Mol. Opt. Phys. 40 S45.

[20]Almeida M P, Melo F, Salles A, et al 2006 Quantum Optics Conference, Pucon, Chile .

[21]Man Z X, Xia Y J and Nguyen B A 2008 J. Phys.B: At. Mol. Opt. Phys. 41085503.

[22]Jaynes E T and Cummings F W 1963 Proc. IEEE 5189.

[23]Boozer A D, Boca A, Miller R, Northup T E and Kimble H J 2006 Phys. Rev. Lett. 97083602.

[24]Fortier K M, Kim S Y, Gibbons M J, Ahmadi and Chapman M S 2007 Phys. Rev. Lett. 98233601.

[25]Vidal G and Werner R F 2002 Phys. Rev. A 65 032314; Audenaert K, Plenio M B and Eisert J 2003

Phys. Rev. Lett. 90027901.

[26]Lee J, Kim M S, Park Y J and Lee S 2000 Journal of Modern Optics 472151.

[27]Coffman V, Kundu J, and Wootters W K 2000 Phys. Rev. A 61052306.

[28]Solano E, Agarwal G S, and Walther H 2003 Phys. Rev. Lett. 90027903.

[29]Haroche S and Raimond J M 2006 Exploring the Quantum (Oxford University Press, Oxford).

[30]Lougovski P, Casagrande F, Lulli A and Solano E 2007 Phys. Rev. A 76033802.

[31]Mundt A B, Kreuter A, Becher C, Leibfried D, Eschner J, Schmidt-Kaler F and Blatt R 2002 Phys. Rev. Lett. 89103001. 